\title{
Perovskite Cells on the Right Track
}

\author{
The semiconductor perovskite is seen as a new hope to bring the production price of solar \\ cells down below that of silicon cells used so far. Empa, Switzerland, is developing new \\ manufacturing processes to make perovskite solar cells not only cheaper but also faster to \\ produce and make them ready for industrial use.
}

Much has changed since the development of the first perovskite solar cell in 2009: Its efficiency is now equal to that of a conventional silicon cell. But it still had some weaknesses in the beginning, as it was very sensitive to moisture, oxygen, heat, UV light and mechanical stress. This made the cell less durable. In 2014, Grätzel and Han found a solution to this problem. They developed a cell with a mesoporous framework of oxides and carbon. But this idea was not yet marketable.

At least until now: Frank Nüesch, Head of Empa's Functional Polymers Department, and his team have been working intensively in recent years on new manufacturing processes for precisely these solar cells in order to produce them not only faster but also cheaper. To this end, the researchers collaborated with Solaronix SA, a company based in Switzerland. Together they produced a functional perovskite cell on a laboratory scale with a surface area of $10 \times 10 \mathrm{~cm}^{2}$.

\section{Slot-die instead of screen printing}

For the production of this novel perovskite cell, the slot-die process is used. Here, the material layer is applied to a substrate of glass and then structured by removing excess material with a laser. "With the new coating process, we can not only coat faster, but also define the thickness of the layers more flexibly," says Nüesch. In the future, the slot-die process will make it possible to coat meterlong webs relatively easily and quickly. The coating speed is then also the central element in a possible industrialization of perovskite cell production.

A total of five layers of different materials, including titanium oxide, zirconia and graph-

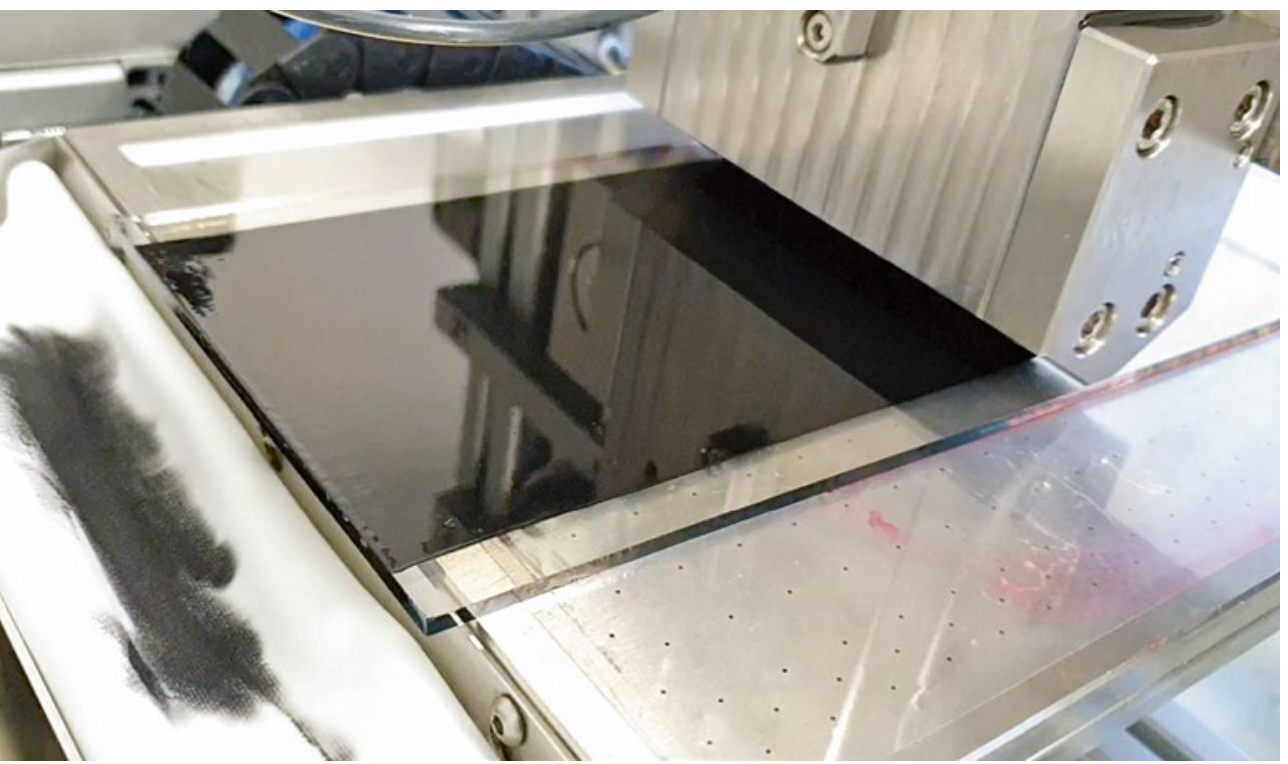

Figure 1 The slot die applies a carbon layer to the glass substrate. This allows all five layers of the solar cell to be applied one after the other and dried together. With the conventional screen printing process, each layer had to be dried separately for at least one hour. (@ Empa)

ite, are required for such a cell. Whereas in the screen printing process used so far, the layers have to be dried and sintered individually, which takes a lot of time and energy, in the slot die process all layers can be applied directly one after the other and sintered together (Figure 1). "With this new process we can print seven times faster than with the previous screen printing method," explains Nüesch. The perovskite solar cell gets its final touch by applying the perovskite absorber by means of inkjet printing, the so-called infiltration. Here the perovskite is no longer applied to the substrate as a solid layer, but seeps through all the porous layers of the solar cell down to the bottom.

\section{A successful cooperation}

In developing the new process, the Empa team worked closely with Solaronix experts. They are the source of the 'inks', nanoscale conductors, semiconductors and insulators, for printing the individual, wafer-thin layers of the solar cell. The difficulty for the Empa researchers was to prepare this ink in such a way that it was suitable for the slot-die process. The various settings of the coating unit, such as the speed of the slot-die, the flow rate and the distance between the slot-die and the substrate, also had to be coordinated in order to achieve an optimum result.

The perovskite solar cells produced using this new process also have longer service life compared to previous perovskite cells. In a next step, field tests will follow: At the end of 2020, the perovskite solar cells will be installed on the Empa campus in Dübendorf, where they will have to prove themselves in everyday use.

Contact:

Empa, Dübendorf, Switzerland, www.empa.ch 